\title{
Necking in 2D incompressible polyconvex materials: theoretical framework and numerical simulations
}

\author{
Carlos Mora-Corral and Magdalena Strugaru
}

April 12, 2017

\begin{abstract}
We show examples of 2D incompressible isotropic homogeneous hyperelastic materials with a polyconvex stored-energy function that present necking. The construction of the stored-energy function of a material satisfying all those properties requires a fine search. We used the software Algencan to perform numerical experiments and visualize necking for the examples constructed. The algorithm is based on minimization of the elastic energy under the nonconvex constraint of incompressibility.
\end{abstract}

\section{Introduction}

Consider a two-dimensional (2D) bar or a three-dimensional (3D) cylinder made of a homogeneous elastic material. When subjected to a uniaxial tension, it usually deforms homogeneously until a critical load is reached. After that, the material ceases to be elastic, or else a bifurcation occurs. This bifurcation phenomenon can be briefly described as follows. One can express the tensile force $S$ on the ends of the body as a function of the elongation $\lambda$. When $S$ is increasing with $\lambda$, no bifurcation occurs, but when $S$ has the profile of increasing-decreasing-increasing, then the homogeneous deformation loses its stability when $\lambda$ crosses the local maximum of $S$. If the material remains in the elastic regime, the bifurcation often occurs in the form of necking, which is the object of this paper.

Necking is a type of deformation that undergo some materials (such as polymers and ductile metals) when subjected to a high tensile extension. It consists of a decrease of the thickness in some regions of the body, called necks. Not all materials present necking, and, in fact, it is at first glance counter-intuitive that necking is energetically more favourable than the homogeneous deformation satisfying the same boundary conditions.

Although necking is truly a plastic phenomenon, since it typically involves an irreversible deformation, the first steps of the necking process can still be modelled using nonlinear elasticity; we prefer the use of nonlinear elasticity since its general modelling is better understood than that of plasticity and does not require ad hoc assumptions. Necking is also an evolutionary process, but in this paper we focus on equilibrium solutions, in fact, minimizers of the elastic energy.

The literature on necking is vast. It has mainly been studied within the framework of plasticity or elasto-plasticity. In the next paragraphs we comment some works that model necking successfully only with nonlinear elasticity and have relevance in our study.

Antman [1] uses rod theory together with a bifurcation analysis to show non-uniqueness of solutions, which can be interpreted as necking. The use of rod theory reduces the analysis to one dimension. Extensions of his analysis can be found in [3].

Owen [30] uses rod theory for compressible and incompressible materials. He employs methods of Calculus of Variations to ascertain the stability of the homogeneous deformation and of the different types of nontrivial solutions that can bifurcate. He recovers the result that if the elongation of the rod is prescribed and the load/extension curve is not increasing, then the homogeneous solution becomes unstable after a critical elongation. 
Mielke [26, Ch. 10] studies a 2D body which is homogeneous, compressible and isotropic. He points out that non-monotonicity of the tensile load (which, as mentioned above, was known to be a condition for bifurcation from the homogeneous deformation) can only occur for non-convex stored-energy functions $W$, but it is perfectly compatible with the ellipticity of the equilibrium equations and, in fact, with polyconvexity of $W$. Using arguments from dynamical systems, and, in particular, center manifolds, he proves the existence of stable solutions with necks.

$\mathrm{Fu}[20, \mathrm{Ch} .10 .4]$ studies an incompressible plate using perturbation theory and his conclusions are similar to $[26$, Ch. 10].

Finally, we comment the works of Sivaloganathan \& Spector [33, 34, 35], which have been a great motivation for our study. They prove that, for $2 \mathrm{D}$ and $3 \mathrm{D}$ compressible or incompressible, homogeneous, isotropic materials, a fairly general family of polyconvex energies yields the homogeneous deformation as the absolute minimizer of the energy. This, in particular, excludes necking and any bifurcation phenomenon. In contrast with previous approaches, they took a global viewpoint: while it was known that those assumptions prohibit bifurcation, this was the first result showing global minimality of the energy. The focus on polyconvex stored-energy functions is motivated by the fact that they constitute the general assumption for the existence of minimizers [6].

To sum up, most of the studies of necking with nonlinear elasticity deal with general sufficient conditions for which necking does not occur $[38,23,16,36]$, or with models for which necking does occur and is stable in the sense of linear stability or local minimality of the energy $[1,3,13,30,26,20,2,15]$. As far as we know, only $[33,34,35]$ study global minimization. They dealt with a fairly general family of homogeneous, isotropic, polyconvex energies. However, they did not exhaust all such energies.

In this paper we take the viewpoint of [33] and complement it by showing that, in $2 \mathrm{D}$, it is possible to construct polyconvex stored-energy functions for incompressible, homogeneous, isotropic materials that do not satisfy the assumptions of [33] and for which the homogeneous deformation is not a global minimizer. Moreover, numerical experiments suggest that necking (or, rather, half-necking) is a global minimizer of the energy.

This paper has both analytic and numeric facets. The analytic part consists in finding an explicit polyconvex stored-energy function for incompressible, homogeneous, isotropic materials that do not satisfy the assumptions of [33]. While in 3D it is easy to find those examples (see $[5,30]$ ), in 2D it turned out to involve a delicate calculation, even though [26, Ch. 10] showed how one can construct such kind of energy in the compressible case. We mention that polyconvexity implies that the homogeneous deformation satisfies all usual necessary conditions (e.g., stationary point, Legendre-Hadamard) for local miminizers [14, Rk. 5.4]. Thus, a global analysis based on minimization is required.

The numerical part consists in developing the finite element implementation (which is chosen, for simplicity, to be piecewise linear) and then to compute minimizers of the elastic energy in the finite element space. For the minimization process we employed Algencan [25, 8], an open-source Fortran routine for solving constrained optimization problems using the augmented Lagrangian principles. In our case, the key constraint used is the incompressibility.

The outline of this paper is as follows. In Section 2 we establish the precise formulation of the problem. Section 3 describes a general method for constructing polyconvex functions $W$ not in the class of [33], and shows a particular one-parameter family of such $W$ of polynomial form. In Section 4 we assume that the deformation is symmetric with respect to the vertical axis and recast the problem accordingly. In Section 5 we present two important initial deformations, which are idealized necks, and explain the equal area rule in this context. In Section 6 we perform the numerical experiments: we will see numerically that the choice of $W$ made before corresponds to a material that, for some values of the elongation, presents necking. This means that it is a necking deformation that is the minimizer of the energy. This answers in the affirmative the $2 \mathrm{D}$ analogue of a question posed in [34, p. 316]: necking is possible for isotropic homogeneous polyconvex materials out of the class of [33]. We present in Section 7 a list of conclusions. 


\section{Setting of the problem}

This section closely follows the setting of [33]. Consider a $2 \mathrm{D}$ bar occupying the region $\Omega:=(-R, R) \times(0, L)$ in the plane $\mathbb{R}^{2}$. A deformation of the bar is represented by a map $\mathbf{u}: \Omega \rightarrow \mathbb{R}^{2}$. We write $\mathbf{u}(\mathbf{x})=$ $\left(u^{1}\left(x_{1}, x_{2}\right), u^{2}\left(x_{1}, x_{2}\right)\right)$ for $\mathbf{x} \in \Omega$ and assume that $\mathbf{u}$ is in the Sobolev space $W^{1, p}$ for some $1<p<\infty$, or else it is of class $C^{1}$ in $\Omega$ and continuous in $[-R, R] \times[0, L]$. This distinction is, in fact, inessential in the analysis of the paper. The body is clamped at the bottom $[-R, R] \times\{0\}$, while at the top $[-R, R] \times\{L\}$ it is stretched so as to have a final height of $\lambda>1$ times the original height. This is expressed in the Dirichlet boundary conditions

$$
u^{2}\left(x_{1}, 0\right)=0 \text { and } u^{2}\left(x_{1}, L\right)=\lambda L \quad \text { for all } x_{1} \in[-R, R]
$$

also called clamped boundary conditions. The sides $\{-R, R\} \times(0, L)$ of the bar are stress-free; this condition need not be imposed explicitly since minimizers of the energy will automatically satisfy that. We assume that the body is incompressible, which is expressed in the nonlinear constraint $\operatorname{det} D \mathbf{u}=1$. Here $D \mathbf{u}$ denotes the deformation gradient. In order to avoid interpenetration of matter, we also impose that $\mathbf{u}$ is injective.

The problem, as stated, is invariant under horizontal translations. In order to remove this invariance, we impose the normalization condition

$$
\int_{\Omega} u^{1}(\mathbf{x}) \mathrm{d} \mathbf{x}=0
$$

also called sliding boundary conditions. Thus, the set of admissible deformations $\mathcal{A}_{\lambda}$ is the set of injective $\mathbf{u}$ in $W^{1, p}$ such that $\operatorname{det} D \mathbf{u}=1$ and (2.1)-(2.2) hold.

Let $W: \mathbb{R}_{1}^{2 \times 2} \rightarrow[0, \infty)$ be the stored-energy function of the material; here $\mathbb{R}_{1}^{2 \times 2}$ denotes the set of $2 \times 2$ matrices with determinant 1 . The fact that $W$ is defined only in $\mathbb{R}_{1}^{2 \times 2}$ is because the material is incompressible. It is also assumed to be isotropic and, naturally, the principle of frame indifference must hold. Therefore, standard representation theorems (see, e.g., $[22,12]$ ) imply that there is a function $h:[\sqrt{2}, \infty) \rightarrow[0, \infty)$ such that

$$
W(\mathbf{F})=h(|\mathbf{F}|), \quad \mathbf{F} \in \mathbb{R}_{1}^{2 \times 2} .
$$

It is well-known (see, e.g., [6]) that if $h$ is convex and increasing then $W$ is polyconvex (see, e.g., [14]), which is an essential assumption for the existence of minimizers. (In this paper, we use words like increasing and positive in the wide sense; we use strictly for the restricted sense). This family of such $h$ forms a fairly general class of polyconvex maps, although it does not exhaust the collection of all polyconvex maps (see, e.g., [31, Th. 3.1] or [32, Th. 4.1]).

The elastic energy of a deformation $\mathbf{u}$ is

$$
E(\mathbf{u}):=\int_{\Omega} W(D \mathbf{u}(\mathbf{x})) \mathrm{d} \mathbf{x} .
$$

The main result of [33] states that if $h$ is convex and increasing then the homogeneous deformation

$$
\mathbf{u}_{H}\left(x_{1}, x_{2}\right):=\left(\lambda^{-1} x_{1}, \lambda x_{2}\right)
$$

is a minimizer of $E$ in $\mathcal{A}_{\lambda}$. This, in particular, excludes any bifurcation branch from the homogeneous solution. As mentioned in [33, Rk. 3.7], their analysis, initially done in the class of $C^{1}$ deformations, is also valid for Sobolev maps $W^{1, p}\left(\Omega, \mathbb{R}^{2}\right)$. See, in addition, [28], where it was proved in the more general setting of $S B V$ maps, thus allowing for fracture.

\section{Incompressible isotropic polyconvex materials with necking}

For each $\mathbf{F} \in \mathbb{R}_{1}^{2 \times 2}$, let $\nu_{1}, \nu_{2}$ be its singular values. Since $\nu_{1} \nu_{2}=1$, its largest singular value, denoted by $\nu(\mathbf{F})$, satisfies $\nu(\mathbf{F}) \geq 1$. The norm of $\mathbf{F}$ is defined as

$$
|\mathbf{F}|:=\left(\sum_{i, j=1}^{2} F_{i j}^{2}\right)^{\frac{1}{2}}
$$


and satisfies

$$
|\mathbf{F}|^{2}=\nu_{1}^{2}+\nu_{2}^{2}=\nu(\mathbf{F})^{2}+\nu(\mathbf{F})^{-2}
$$

Therefore, when we define $g:[\sqrt{2}, \infty) \rightarrow[1, \infty)$ as

$$
g(s):=\sqrt{\frac{s^{2}+\sqrt{s^{4}-4}}{2}},
$$

we have that $g^{-1}(t)=\sqrt{t^{2}+t^{-2}},|\mathbf{F}|=g^{-1}(\nu(\mathbf{F}))$ and $\nu(\mathbf{F})=g(|\mathbf{F}|)$.

Let $W: \mathbb{R}_{1}^{2 \times 2} \rightarrow \mathbb{R}$ be the stored-energy function of the material, which is assumed to be homogeneous, isotropic and incompressible. As mentioned in Section $2, W$ depends only on $|\mathbf{F}|$ or, equivalently, on $\nu(\mathbf{F})$ or on $|\mathbf{F}|^{2}$. Hence there exist

$$
\varphi:[1, \infty) \rightarrow \mathbb{R}, \quad h:[\sqrt{2}, \infty) \rightarrow \mathbb{R} \quad \text { and } \quad H:[2, \infty) \rightarrow \mathbb{R}
$$

such that $W(\mathbf{F})=\varphi(\nu(\mathbf{F}))=h(|\mathbf{F}|)=H\left(|\mathbf{F}|^{2}\right)$. Naturally, $h=\varphi \circ g=H \circ Q$ with $Q:[\sqrt{2}, \infty) \rightarrow[2, \infty)$, $Q(t):=t^{2}$. It is also customary to express $W$ as a symmetric function $\Phi$ of the singular values $\nu_{1}, \nu_{2}$ of $\mathbf{F}$. Since $\varphi(s)=H\left(s^{2}+s^{-2}\right)$ for all $s \geq 1$, we can take

$$
\Phi:(0, \infty) \times(0, \infty) \rightarrow \mathbb{R}, \quad \Phi\left(\nu_{1}, \nu_{2}\right)=H\left(\nu_{1}^{2}+\nu_{2}^{2}\right),
$$

so that $\varphi(s)=\Phi\left(s, s^{-1}\right)$.

The relationship between the differentiability properties of the functions $W, \varphi, h, H, \Phi$ is tricky [7]. Nevertheless, we know from $[7,37]$ that $W$ is of class $C^{r}$ in $\mathbb{R}_{+}^{2 \times 2}$ (the set of matrices with positive determinant) if and only if $\Phi$ is of class $C^{r}$ in $(0, \infty)^{2}$, for $r \in \mathbb{N} \cup\{\infty\}$. Since we will deal with convexity assumptions (for which second derivatives play a prominent role), we assume from now on that $W$ admits a $C^{2}$ extension to $\mathbb{R}_{+}^{2 \times 2}$. Therefore, $\Phi$ and $\varphi$ are of class $C^{2}$.

It is easily seen that

$$
\varphi^{\prime}(1)=0 \quad \text { and } \quad \varphi^{\prime \prime}(1)=2\left(\frac{\partial^{2} \Phi}{\partial \nu_{1}^{2}}(1,1)-\frac{\partial^{2} \Phi}{\partial \nu_{1} \partial \nu_{2}}(1,1)+\frac{\partial \Phi}{\partial \nu_{1}}(1,1)\right) .
$$

In order to find out the linearly elastic behaviour of the material, as the material is isotropic, it is enough to calculate the two Lamé parameters $\lambda$ and $\mu$. In fact, since the material is incompressible, only the Lamé parameter $\mu$ is relevant. Using (3.1) and formula [29, eq. (6.1.88)] for the Lamé parameter, it can be seen that the identity is stress-free and

$$
\mu=\frac{1}{4} \varphi^{\prime \prime}(1)
$$

Recall $[6,14]$ that, in general, a function defined in the set of matrices $\mathbf{F} \in \mathbb{R}^{2 \times 2}$ is polyconvex if it can be expressed as a convex function of $\mathbf{F}$ and $\operatorname{det} \mathbf{F}$. Characterizations of polyconvexity for isotropic materials can be found in $[31,32]$. In the presence of incompressibility, the corresponding result was proved by Mielke [27, Th. 5.1] and reads as follows.

Proposition 3.1. Define $A:[0, \infty) \rightarrow[1, \infty)$ as

$$
A(\rho):=\frac{\rho}{2}+\sqrt{1+\frac{\rho^{2}}{4}} .
$$

Then, $W$ is polyconvex if and only if $\varphi \circ A$ is increasing and convex.

It is known that, in general, polyconvexity is not a local condition [24]. Nevertheless, in dimension 2 and in the incompressible case, Proposition 3.1 shows in particular that it is a local condition. In fact, we can easily express a necessary and sufficient condition for polyconvexity in terms of the positivity of certain functions. 
Lemma 3.2. Assume $W$ is of class $C^{2}$ in $\mathbb{R}_{+}^{2 \times 2}$. Then, $W$ is polyconvex if and only if

$$
\varphi^{\prime}(t) \geq 0 \quad \text { and } \quad t\left(1+t^{2}\right) \varphi^{\prime \prime}(t)+2 \varphi^{\prime}(t) \geq 0 \quad \forall t \in[1, \infty) .
$$

Proof. As $A$ is strictly increasing, we have that $\varphi \circ A$ is increasing if and only if so is $\varphi$, hence this is equivalent to the inequality $\varphi^{\prime}(t) \geq 0$ for all $t \geq 1$.

Now, $\varphi \circ A$ is convex if and only if any of the equivalent conditions of the following chain holds:

- $(\varphi \circ A)^{\prime \prime} \geq 0$ in $[0, \infty)$.

- $\left(\varphi^{\prime \prime} \circ A\right)\left(A^{\prime}\right)^{2}+\left(\varphi^{\prime} \circ A\right) A^{\prime \prime} \geq 0$ in $[0, \infty)$.

- $\varphi^{\prime \prime}\left(A^{\prime} \circ A^{-1}\right)^{2}+\varphi^{\prime}\left(A^{\prime \prime} \circ A^{-1}\right) \geq 0$ in $[1, \infty)$.

Having in mind that

$$
A^{-1}(t)=t-\frac{1}{t}, \quad\left(A^{\prime} \circ A^{-1}\right)(t)=\frac{t^{2}}{1+t^{2}}, \quad\left(A^{\prime \prime} \circ A^{-1}\right)(t)=\frac{2 t^{3}}{\left(1+t^{2}\right)^{3}}
$$

for $t \geq 1$, the chain of equivalences continues as $t\left(1+t^{2}\right) \varphi^{\prime \prime}(t)+2 \varphi^{\prime}(t) \geq 0$.

As mentioned in Section 2, Sivaloganathan \& Spector [33] worked with the assumption that $h$ is increasing and convex, which is a sufficient condition for the polyconvexity of $W$. As in Lemma 3.2, we can find a necessary and sufficient condition for this assumption.

Lemma 3.3. Assume $W$ is of class $C^{2}$ in $\mathbb{R}_{+}^{2 \times 2}$. Then, $h$ is increasing and convex if and only if

$$
\varphi^{\prime}(t) \geq 0 \quad \text { and } \quad t\left(t^{8}-1\right) \varphi^{\prime \prime}(t)-2\left(1+3 t^{4}\right) \varphi^{\prime}(t) \geq 0 \quad \forall t \in[1, \infty) .
$$

Proof. As $g$ is strictly increasing, we have that $h$ is increasing if and only if so is $\varphi$, whence we have the equivalent condition $\varphi^{\prime} \geq 0$ in $[1, \infty)$.

Now, $h$ is convex if and only if any of the equivalent conditions of the following chain holds:

- $(\varphi \circ g)^{\prime \prime} \geq 0$ in $[\sqrt{2}, \infty)$.

- $\varphi^{\prime \prime}\left(g^{\prime} \circ g^{-1}\right)^{2}+\varphi^{\prime}\left(g^{\prime \prime} \circ g^{-1}\right) \geq 0$ in $[1, \infty)$.

Having in mind that

$$
\left(g^{\prime} \circ g^{-1}\right)(t)^{2}=t^{4} \frac{1+t^{4}}{\left(t^{4}-1\right)^{2}}, \quad\left(g^{\prime \prime} \circ g^{-1}\right)(t)=-2 t^{3} \frac{1+3 t^{4}}{\left(t^{4}-1\right)^{3}}
$$

for $t \geq 1$, the chain of equivalences continues as $t\left(t^{8}-1\right) \varphi^{\prime \prime}(t)-2\left(1+3 t^{4}\right) \varphi^{\prime}(t) \geq 0$.

It is apparent that there are functions $\varphi$ that satisfy the conditions of Lemma 3.2 but not of Lemma 3.3. However, writing down an explicit example does not seem immediate.

Now we recall a result on bifurcation, mentioned in [33, Rk. 3.8]. Let $S_{1}$ be the lateral force on the rectangle and $S_{2}$ the force on the top and bottom. It was shown by Hill \& Hutchinson [23] (see also $[16,36,17]$ ) that if the force on the boundary (in this case, $S_{2}$ ) increases with the extension then there is no bifurcation from the homogeneous deformation. Having in mind the familiar stress-stretch relations

$$
S_{1}=\frac{\partial \Phi}{\partial \nu_{1}}-P \nu_{1}^{-1}, \quad S_{2}=\frac{\partial \Phi}{\partial \nu_{2}}-P \nu_{2}^{-1},
$$

where $P$ is the Lagrange multiplier associated with the restriction of incompressibility, and the fact that $S_{1}=0$ (because of the boundary conditions on the lateral surface), we obtain, using the representation $\varphi(\nu)=\Phi\left(\nu, \nu^{-1}\right)$, that $S_{2}(\nu)=\varphi^{\prime}(\nu)$. Thus, $S_{2}$ is increasing if and only if $\varphi^{\prime \prime} \geq 0$. With this and Lemma 3.3 , we obtain an alternative derivation to that of [33] of the fact that if $h$ is increasing and convex then $S_{2}$ is 
increasing with $\nu$. In addition, one recovers a well-known heuristic rule according to which necking appears when $\varphi^{\prime}$ is not always increasing; in particular, when it has the profile of increasing-decreasing-increasing. This leads to the equal area rule (see, e.g., [18]), which will be further explained in Section 5.

We are interested in functions that satisfy the conditions of Lemma 3.2 but not of Lemma 3.3. In our search of necking, instead of negating the condition of Lemma 3.3, in view of the bifurcation result above, we will impose the stronger condition that $\varphi$ is not convex, i.e., $\varphi^{\prime \prime}(t)<0$ some $t$.

We additionally impose to the energy the normalization conditions $W \geq 0$ and $W(\mathbf{I})=0$. In terms of $\varphi$, they read as $\varphi \geq 0$ and $\varphi(1)=0$. In fact, conditions $\varphi(1)=0$ and $\varphi^{\prime} \geq 0$ (see Lemma 3.2) imply $\varphi \geq 0$. To sum up, we look for functions $\varphi:[1, \infty) \rightarrow \mathbb{R}$ of class $C^{2}$ such that

(C1) $\varphi(1)=0$ and $\varphi^{\prime}(1)=0$.

(C2) $\varphi^{\prime}(t) \geq 0$ and $t\left(1+t^{2}\right) \varphi^{\prime \prime}(t)+2 \varphi^{\prime}(t) \geq 0$ for all $t \in[1, \infty)$.

(C3) $\varphi^{\prime \prime}(t)<0$ for some $t \in(1, \infty)$.

As pointed out before, conditions (C1)-(C3) are local, so a useful way of defining $\varphi$ is piecewise. For the modelling of actual materials, we recommend to define $\varphi$ in three polynomial pieces:

- For values of $t$ near 1, to meet the linearly elastic behaviour of the material (see (3.2)).

- For middle values of $t$, to make the profile of $\varphi^{\prime}$ of increasing-decreasing-increasing and, hence, to model the necking behaviour.

- For large values of $t$, to meet the behaviour of $W$ for large strains.

For the sake of simplicity of an already complex argument, we chose, nevertheless, $\varphi$ to be a polynomial, since there are standard algorithms (for example, those based on Sturm's theorem) deciding the positivity of a polynomial in a given interval.

First, it is easy to check that no cubic polynomial $\varphi$ can satisfy $(\mathrm{C} 1)-(\mathrm{C} 3)$ simultaneously. We then try a quartic polynomial. In order to ensure $(\mathrm{C} 1)$, we express $\varphi$ in the form

$$
\varphi(t)=b(t-1)^{2}+c(t-1)^{3}+\frac{1}{4}(t-1)^{4} .
$$

for some $b, c \in \mathbb{R}$. The coefficient $\frac{1}{4}$ in the factor $(t-1)^{4}$ is just a normalization condition. We used the software Mathematica ${ }^{\circledR}$ to find out when $(\mathrm{C} 2)-(\mathrm{C} 3)$ hold. A handmade proof can be done, nevertheless, with the use of Sturm's polynomials.

Proposition 3.4. The polynomial (3.3) satisfies (C1)-(C3) if and only if

$$
b>0 \quad \text { and } \quad F(b) \leq c<-\sqrt{\frac{2 b}{3}},
$$

where $F(b)$ is the first root of the polynomial in $x$

$$
\begin{aligned}
& -206 b+284 b^{2}-2738 b^{3}+152 b^{4}-8 b^{5}+\left(1398 b+2730 b^{2}+1692 b^{3}+96 b^{4}\right) x \\
& +\left(309-4686 b+1728 b^{2}-1440 b^{3}+12 b^{4}\right) x^{2}+\left(-1994-852 b-1072 b^{2}-144 b^{3}\right) x^{3} \\
& +\left(6000+1320 b+1800 b^{2}\right) x^{4}+(-4272-2064 b) x^{5}+2124 x^{6} .
\end{aligned}
$$

Moreover, $F(b)<-\sqrt{2 b / 3}$ and $F^{\prime}(b)<0$ for all $b>0$.

Even though $F(b)<-\sqrt{2 b / 3}$ for all $b>0$, the difference $-\sqrt{2 b / 3}-F(b)$ is small, so, for a given $b>0$, the range of $c$ meeting the conditions of Proposition 3.4 is narrow; this comes as no surprise since conditions $(\mathrm{C} 2)$ and (C3) are somewhat competing. In Figure 1 we plot the graphs of $F(b)$ and $-\sqrt{2 b / 3}$, together with another function $G(b)$ to be described below. 


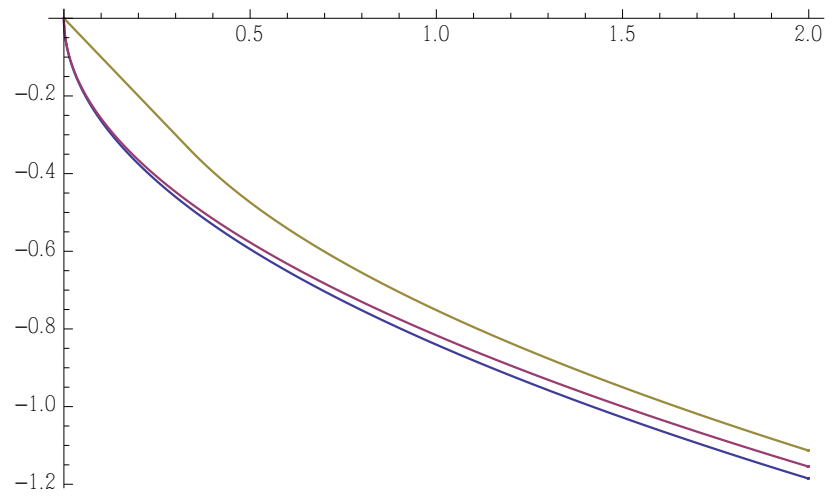

Figure 1: Plot of $F(b)$ (blue, below), $-\sqrt{2 b / 3}$ (magenta, middle) and $G(b)$ (yellow, above) for $0 \leq b \leq 2$.
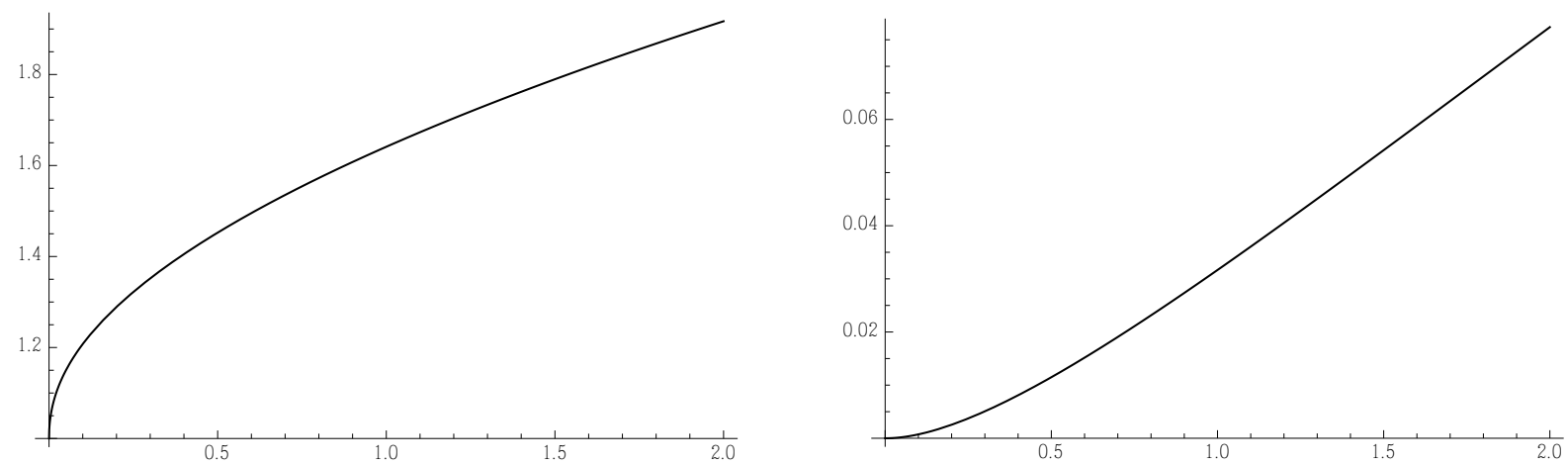

Figure 2: Plot of $p_{-}$(left) and $\varphi^{\prime}\left(p_{-}\right)-\varphi^{\prime}\left(p_{+}\right)$(right) for $0 \leq b \leq 2$.

The fact that $b>0$ has no other restriction shows, thanks to (3.2), that necking is compatible with any linear behaviour of the material, as showed in Mielke [26, Sect. 10.2] in the compressible case.

The graph of $\varphi^{\prime}$ for the values of Proposition 3.4 has the typical increasing-decreasing-increasing profile of a cubic polynomial; see Figure 3. An important parameter for the necking process is the well depth, i.e., the difference between the local maximum of $\varphi^{\prime}$ and its local minimum (this is again related to the equal area rule). The two critical points of $\varphi^{\prime}$ are located at

$$
p_{ \pm}:=1-c \pm \sqrt{c^{2}-\frac{2 b}{3}}
$$

so the well depth equals

$$
\varphi^{\prime}\left(p_{-}\right)-\varphi^{\prime}\left(p_{+}\right)=\frac{4\left(-2 b+3 c^{2}\right)^{\frac{3}{2}}}{3 \sqrt{3}} .
$$

For a given $b>0$, its maximum is achieved when $-2 b+3 c^{2}$ is maximum, i.e., when $c=F(b)$. Mathematica ${ }^{\circledR}$ shows that the function $b \mapsto-2 b+3 F(b)^{2}$ is increasing. On the other hand, $p_{-}$increases with $b$ and decreases with $c$; therefore, it also increases with $b$ when $c$ is evaluated at $F(b)$. Consequently, even though the bigger the $b$, the deeper the well and the more visible the necking, the value of $b$ has to be limited since necking will only appear after the extension crosses $p_{-}$. A big value of $p_{-}$would mean that an unrealistically large extension of the material (higher, for example, than that needed to break it) would be necessary to produce necking. In Figure 2 we show the plots of $p_{-}$and of the well depth $\varphi^{\prime}\left(p_{-}\right)-\varphi^{\prime}\left(p_{+}\right)$evaluated at $c=F(b)$, for $0 \leq b \leq 2$. These graphs will help us to make a suitable choice of the parameters when we perform the numerical experiments in Section 6. 
We are also interested in the case when the conditions of Lemma 3.2 hold, those of Lemma 3.3 do not hold, and (C3) does not hold either. In this case, in principle, anything can happen: the minimizer may be the homogeneous deformation or a sort of necking or any other instability. This is not in contradiction with the results of $[23,16,36,17]$ mentioned above, according to which the condition $\varphi^{\prime \prime} \geq 0$ excludes bifurcation from $\mathbf{u}_{H}$, since it is theoretically possible that the absolute minimizer does not bifurcate from $\mathbf{u}_{H}$. This global analysis is, in fact, one of the goals of this work. This situation (the conditions of Lemma 3.2 hold but not those of Lemma 3.3 or (C3)) corresponds to the assumptions that $W$ is polyconvex, $h$ is not convex and $\varphi$ is convex. In terms of $\varphi$, this reads as

$$
\varphi^{\prime \prime}(t) \geq 0 \quad \text { for all } t \in[1, \infty), \quad \text { and } \quad t\left(t^{8}-1\right) \varphi^{\prime \prime}(t)-2\left(1+3 t^{4}\right) \varphi^{\prime}(t)<0 \quad \text { for some } t \in(1, \infty) .
$$

In terms of the coefficients $b, c$ of $(3.3)$, Mathematica ${ }^{\circledR}$ found this to be equivalent to

$$
b>0 \text { and }-\sqrt{\frac{2 b}{3}} \leq c<G(b),
$$

for a certain function $G$ whose explicit expression is not important here; its graph is shown in Figure 1. As before, given $b>0$, we choose $c$ to be the lower limit, so that condition

$$
t\left(t^{8}-1\right) \varphi^{\prime \prime}(t)-2\left(1+3 t^{4}\right) \varphi^{\prime}(t) \geq 0 \quad \forall t \in[1, \infty)
$$

is violated "as much as possible". Hence, we take $c=-\sqrt{2 b / 3}$.

We finish this section with a comment on how the expression of $W$ is actually implemented in the code. Among the equivalent representations of $W$ described at the beginning of this section, for the experiments we chose $W(\mathbf{F})=H\left(|\mathbf{F}|^{2}\right)$, since the computation of the square of the norm of a matrix is faster than that of its maximum singular value. However, as we have just seen, the search of a polyconvex stored-energy function possibly giving rise to necking heavily depends on the representation $\varphi$. As shown here, $H=\varphi \circ g \circ Q^{-1}$; in other words, $H=\varphi \circ f$ with $f:=g \circ Q^{-1}:[2, \infty) \rightarrow[1, \infty)$, which is given by

$$
f(s)=\sqrt{\frac{s+\sqrt{s^{2}-4}}{2}} .
$$

The function $H$ is of class $C^{1}$ in $[2, \infty)$, although it is not twice differentiable at 2. More serious than the latter fact is that, in the iterative algorithm of minimization, the constraint $|\mathbf{F}|^{2} \geq 2$ can be violated for the local deformation gradient. Therefore, it is imperative to extend $H$ and its derivatives for values below 2 . The precise form of the extension is not crucial, since in the final deformation all constraints will be satisfied, or at least violated by a tiny quantity. We choose the symmetric extension $\tilde{H}$ of $H$ :

$$
\tilde{H}(s):= \begin{cases}H(s) & \text { if } s \geq 2, \\ H(4-s) & \text { if } s<2 .\end{cases}
$$

Even though $H$ is of class $C^{1}$ in $[2, \infty)$, in the expression of $H^{\prime}$ there appears a denominator that vanishes at 2. To avoid the numerical instabilities coming from this fact, we modified $H^{\prime}$ and $H^{\prime \prime}$ in a small interval $(2-\varepsilon, 2+\varepsilon)$ by declaring them to be the first and second derivative, respectively, of $\varphi(\sqrt{s / 2})$; in other words, we set $\sqrt{s^{2}-4}=0$ in the expression of $f$ when $|s-2|<\varepsilon$. Hence we took as modifications of the derivatives of $\tilde{H}$

$$
\widetilde{H^{\prime}}(s):= \begin{cases}H^{\prime}(s) & \text { if } s \geq 2+\varepsilon, \\ \varphi^{\prime}\left(\sqrt{\frac{s}{2}}\right) \frac{1}{2 \sqrt{2 s}} & \text { if } 2-\varepsilon<s<2+\varepsilon, \\ -H^{\prime}(4-s) & \text { if } s \leq 2-\varepsilon\end{cases}
$$

and

$$
\widetilde{H^{\prime \prime}}(s):= \begin{cases}H^{\prime \prime}(s) & \text { if } s \geq 2+\varepsilon \\ \varphi^{\prime \prime}\left(\sqrt{\frac{s}{2}}\right) \frac{1}{8 s}-\varphi^{\prime}\left(\sqrt{\frac{s}{2}}\right) \frac{1}{4 \sqrt{2 s^{3}}} & \text { if } 2-\varepsilon<s<2+\varepsilon \\ H^{\prime \prime}(4-s) & \text { if } s \leq 2\end{cases}
$$




\section{$4 \quad$ Symmetry}

Recall from Section 2 that our problem is, for a given $\lambda>1$, to minimize the elastic energy $E$ of (2.3) in the class $\mathcal{A}_{\lambda}$ of injective $\mathbf{u}:(-R, R) \times(0, L) \rightarrow \mathbb{R}^{2}$ in $W^{1, p}$ such that $\operatorname{det} D \mathbf{u}=1$ and (2.1)-(2.2) hold.

In the absence of the condition of Lemma 3.3, proving that minimizers are symmetric with respect to the vertical axis turns out to be a difficult task, as suggested by [33], who do it under the assumptions of Lemma 3.3. Nevertheless, our intuition and numerical and actual experiments suggest that the solution is indeed symmetric, and, in particular, that a minimizer $\mathbf{u}$ satisfies $u^{1}\left(0, x_{2}\right)=0$ for all $x_{2} \in[0, L]$. A partial result in this direction is the following.

Proposition 4.1. Let $\mathbf{u}_{0}$ be a minimizer of $E$ in $\mathcal{A}_{\lambda}$ with $u_{0}^{1}\left(0, x_{2}\right)=0$ for all $x_{2} \in(0, L)$. Then:

1) $\int_{(-R, 0) \times(0, L)} W\left(D \mathbf{u}_{0}\right) \mathrm{d} \mathbf{x}=\int_{(0, R) \times(0, L)} W\left(D \mathbf{u}_{0}\right) \mathrm{d} \mathbf{x}$.

2) $\left.\mathbf{u}_{0}\right|_{(-R, 0) \times(0, L)}$ is a minimizer of

$$
\int_{(-R, 0) \times(0, L)} W(D \mathbf{u}) \mathrm{d} \mathbf{x}
$$

under the boundary conditions

$$
u^{1}\left(0, x_{2}\right)=0 \quad \forall x_{2} \in[0, L], \quad u^{2}\left(x_{1}, 0\right)=0 \quad \forall x_{1} \in[-R, 0], \quad u^{2}\left(x_{1}, L\right)=\lambda L \quad \forall x_{1} \in[-R, 0],
$$

and $\left.\mathbf{u}_{0}\right|_{(0, R) \times(0, L)}$ is a minimizer of

$$
\int_{(0, R) \times(0, L)} W(D \mathbf{u}) \mathrm{d} \mathbf{x}
$$

under the boundary conditions

$$
u^{1}\left(0, x_{2}\right)=0 \quad \forall x_{2} \in[0, L], \quad u^{2}\left(x_{1}, 0\right)=0 \quad \forall x_{1} \in[0, R], \quad u^{2}\left(x_{1}, L\right)=\lambda L \quad \forall x_{1} \in[0, R] .
$$

3) The deformations

$$
\mathbf{u}_{1}(\mathbf{x}):= \begin{cases}\mathbf{u}_{0}(\mathbf{x}) & \text { if } x_{1} \geq 0 \\ \left(-u_{0}^{1}\left(-x_{1}, x_{2}\right), u_{0}^{2}\left(-x_{1}, x_{2}\right)\right) & \text { if } x_{1}<0\end{cases}
$$

and

$$
\mathbf{u}_{2}(\mathbf{x}):= \begin{cases}\mathbf{u}_{0}(\mathbf{x}) & \text { if } x_{1} \leq 0 \\ \left(-u_{0}^{1}\left(-x_{1}, x_{2}\right), u_{0}^{2}\left(-x_{1}, x_{2}\right)\right) & \text { if } x_{1}>0\end{cases}
$$

are minimizers of $E$ under the conditions

$$
u^{1}\left(0, x_{2}\right)=0 \quad \forall x_{2} \in[0, L], \quad u^{2}\left(x_{1}, 0\right)=0 \quad \forall x_{1} \in[-R, R], \quad u^{2}\left(x_{1}, L\right)=\lambda L \quad \forall x_{1} \in[-R, R] .
$$

Proof. If the equality in 1) did not hold, then one of the deformations $\mathbf{u}_{1}$ or $\mathbf{u}_{2}$ would have less energy than $\mathbf{u}_{0}$, thus contradicting the minimality of $\mathbf{u}_{0}$. If $\left.\mathbf{u}_{0}\right|_{(-R, 0) \times(0, L)}$ or $\left.\mathbf{u}_{0}\right|_{(0, R) \times(0, L)}$ were not a minimizer of their respective problems in 2), then the extension to $(-R, R) \times(0, L)$ of the minimizers by symmetrization as in $\mathbf{u}_{1}$ or $\mathbf{u}_{2}$ would have less energy than $\mathbf{u}_{0}$, thus contradicting the minimality of $\mathbf{u}_{0}$. Finally, $E\left(\mathbf{u}_{1}\right)=E\left(\mathbf{u}_{2}\right)=$ $E\left(\mathbf{u}_{0}\right)$, so $\mathbf{u}_{1}$ and $\mathbf{u}_{2}$ are minimizers of $E$ under the conditions of 3$)$.

Since in this paper we are not concerned with asymmetries with respect to the vertical axis, but rather with the asymmetries of the necking instability, we assume that the minimizer is symmetric with respect to the vertical axis, in its equivalent form that

$$
u^{1}\left(0, x_{2}\right)=0, \quad \text { for all } x_{2} \in[0, L] .
$$


Now recall that condition (2.2) was added to avoid the trivial non-uniqueness given by translations $\mathbf{u} \rightsquigarrow$ $\mathbf{u}+(a, 0)$. Condition (4.1) also prevents that translation-invariance, so with (4.1), condition (2.2) is not needed any more.

Therefore, from now on we redefine the domain $\Omega$ as $(0, R) \times(0, L)$, the elastic energy as

$$
E(\mathbf{u}):=\int_{(0, R) \times(0, L)} W(D \mathbf{u}(\mathbf{x})) \mathrm{d} \mathbf{x},
$$

and the admissible set $\mathcal{A}_{\lambda}$ as the set of injective $\mathbf{u}:(0, R) \times(0, L) \rightarrow \mathbb{R}^{2}$ in $W^{1, p}$ such that $\operatorname{det} D \mathbf{u}=1$ and

$$
u^{2}\left(x_{1}, 0\right)=0 \text { and } u^{2}\left(x_{1}, L\right)=\lambda L \quad \forall x_{1} \in[0, R], \quad u^{1}\left(0, x_{2}\right)=0 \quad \forall x_{2} \in[0, L] .
$$

They are clamped boundary conditions and the right side of the bar is left free.

\section{Half-necked deformation and the equal area rule}

Apart from the homogeneous deformation $\mathbf{u}_{H}$ of (2.4), we describe another deformation that plays a prominent role in the analysis. It is the idealized half-necked deformation

$$
\mathbf{u}_{N}\left(x_{1}, x_{2}\right):= \begin{cases}\left(\lambda_{1}^{-1} x_{1}, \lambda_{1} x_{2}\right) & \text { if } 0 \leq x_{2} \leq \frac{L\left(\lambda_{2}-\lambda\right)}{\lambda_{2}-\lambda_{1}}, \\ \left(\lambda_{2}^{-1} x_{1}, \lambda_{2} x_{2}-L\left(\lambda_{2}-\lambda\right)\right) & \text { if } \frac{L\left(\lambda_{2}-\lambda\right)}{\lambda_{2}-\lambda_{1}}<x_{2}<L,\end{cases}
$$

for some specific $\lambda_{1}, \lambda_{2}$ with $1 \leq \lambda_{1}<\lambda<\lambda_{2}$. This deformation has the disadvantage of being discontinuous (in particular, it is not in the admissible set $\mathcal{A}_{\lambda}$ ), but otherwise it is immediate to implement, no preliminary calculations are necessary (except for the computation of the actual values of $\lambda_{1}$ and $\lambda_{2}$, see below) and captures the main features of a half-necking: it is incompressible, injective, meets the boundary conditions (4.3) and has two different widths: $R / \lambda_{1}$ and $R / \lambda_{2}$. When $\mathbf{u}_{N}$ is discretized in the finite-element space and thus converted to a piecewise affine function, it becomes continuous: the triangles that lie both in $x_{2} \leq \frac{L\left(\lambda_{2}-\lambda\right)}{\lambda_{2}-\lambda_{1}}$ and in $x_{2}>\frac{L\left(\lambda_{2}-\lambda\right)}{\lambda_{2}-\lambda_{1}}$ automatically generate a transition layer. However, the incompressibility is lost in those triangles.

The height $\frac{L\left(\lambda_{2}-\lambda\right)}{\lambda_{2}-\lambda_{1}}$ at which the interface occurs is chosen so that $u_{N}^{2}$ is continuous; of course, $u_{N}^{1}$ cannot be continuous. The values of $\lambda_{1}, \lambda_{2}$ can be chosen, in principle, arbitrarily. Nevertheless, if we ignore the discontinuity of $u_{N}^{1}$ and, hence, the interfacial energy produced by a smoothed version of the deformation, the energy $E$ (see (4.2)) of $\mathbf{u}_{N}$ is easily shown to be

$$
R \frac{\lambda_{2}-\lambda}{\lambda_{2}-\lambda_{1}} L \varphi\left(\lambda_{1}\right)+R \frac{\lambda-\lambda_{1}}{\lambda_{2}-\lambda_{1}} L \varphi\left(\lambda_{2}\right) .
$$

Elementary arguments show that, if $\varphi$ is not convex in $\left[\lambda_{1}, \lambda_{2}\right]$, then minimizers $\lambda_{1}, \lambda_{2}$ of (5.1) must satisfy

$$
\varphi^{\prime}\left(\lambda_{1}\right)=\varphi^{\prime}\left(\lambda_{2}\right)=\frac{\varphi\left(\lambda_{2}\right)-\varphi\left(\lambda_{1}\right)}{\lambda_{2}-\lambda_{1}} .
$$

If $\varphi$ is convex then the infimum of (5.1) corresponds to $\lambda_{1} \nearrow \lambda$ and $\lambda_{2} \searrow \lambda$, which is the trivial case. This is a further reason to choose a non-convex $\varphi$. Equations (5.2) express the equal area rule (see Figure 3), first introduced by Maxwell and widely used in the van der Waals' theory of phase transitions in fluids (see, e.g., [10]); note that $1<\lambda_{1}<p_{-}<\lambda<p_{+}<\lambda_{2}$. This rule has also been used in the theory of necking (see, e.g., $[18,30,11,19])$. Moreover, Owen [30, Th. 5.1] showed that, for this choice of $\lambda_{1}, \lambda_{2}$, the deformation $\mathbf{u}_{N}$ provides the limit profile of the minimizing deformation of a scaled bar as the length of the bar tends to infinity.

Besides a half-necked deformation, we can also define a center-necked deformation:

$$
\mathbf{u}_{C}\left(x_{1}, x_{2}\right):= \begin{cases}\left(\lambda_{1}^{-1} x_{1}, \lambda_{1} x_{2}\right) & \text { if } 0 \leq x_{2} \leq \frac{\lambda_{2}-\lambda}{\lambda_{2}-\lambda_{1}} \frac{L}{2} \\ \left(\lambda_{2}^{-1} x_{1}, \lambda_{2} x_{2}\right)-\left(0,\left(\lambda_{2}-\lambda\right) \frac{L}{2}\right) & \text { if } \frac{\lambda_{2}-\lambda}{\lambda_{2}-\lambda_{1}} \frac{L}{2}<x_{2}<\frac{\lambda_{2}-2 \lambda_{1}+\lambda}{\lambda_{2}-\lambda_{1}} \frac{L}{2} \\ \left(\lambda_{1}^{-1} x_{1}, \lambda_{1} x_{2}\right)+\left(0,\left(\lambda-\lambda_{1}\right) L\right) & \text { if } \frac{\lambda_{2}-2 \lambda_{1}+\lambda}{\lambda_{2}-\lambda_{1}} \frac{L}{2} \leq x_{2} \leq L\end{cases}
$$



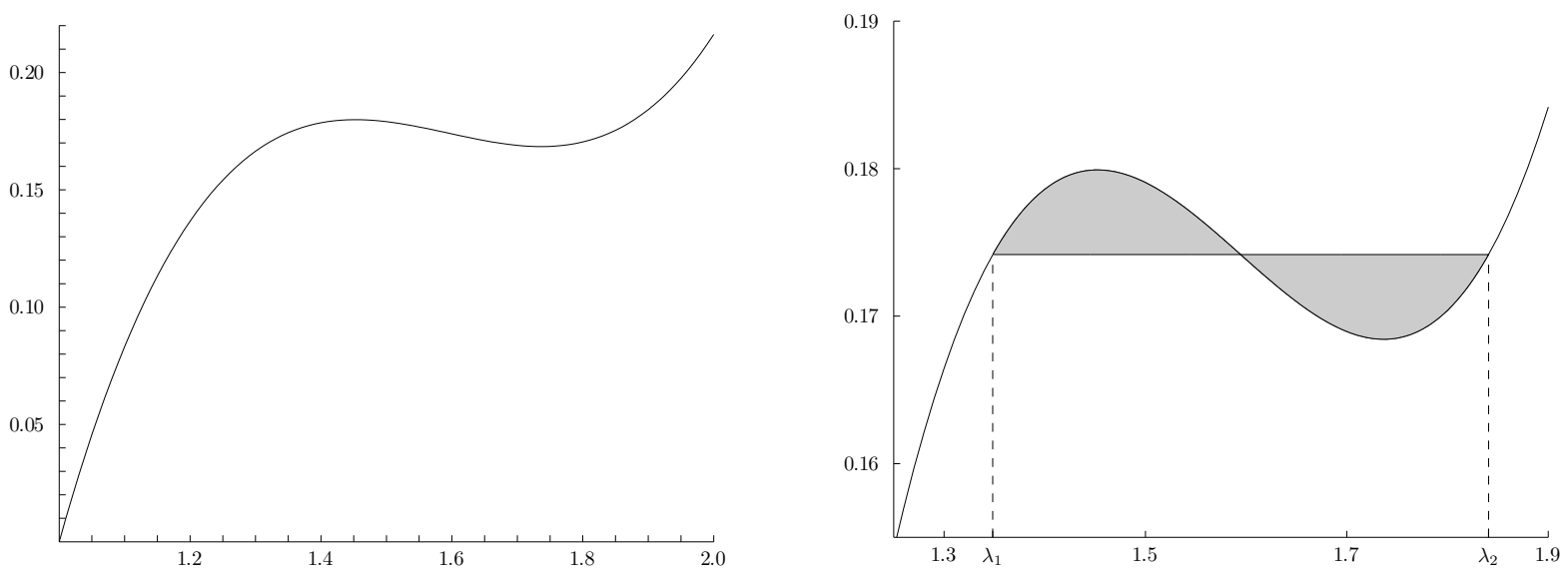

Figure 3: Left: Graph of $\varphi^{\prime}$ for $b=0.5$ and $c=F(b)$. Right: Zoom of the graph showing the choice of $\lambda_{1}$ and $\lambda_{2}$ according to the equal area rule: the area of the graph above the horizontal line equals the area of the graph below it.

which shares many features with $\mathbf{u}_{N}: u_{C}^{2}$ is continuous but $u_{C}^{1}$ is discontinuous, $\mathbf{u}_{C}$ it is not in $\mathcal{A}_{\lambda}$ but it is incompressible, injective, satisfies the boundary conditions (4.3) and has two different widths. Moreover, its energy $E$, if we ignore the interfacial energy induced by the two discontinuity lines, also equals (5.1). However, since the formal computation leading to (5.1) does not take into account energy of the transition layer that a continuous (or Sobolev) deformation must have between the different pieces, it is expected that a smoothed version of $\mathbf{u}_{N}$ has less energy than that of $\mathbf{u}_{C}$, since the former has one interface and the latter has two, while the energy out of the transition regions is the same. We remark that our boundary conditions (4.3) allow for different thicknesses on the top and on the bottom. As a matter of fact, Owen [30, Th. 4.3] showed that, in his rod model, deformations with full necks (as a smoothed version of $\mathbf{u}_{C}$ ) are not even local minimizers, but his boundary conditions are different.

\section{$6 \quad$ Numerical results}

Recall that we aim to minimize (4.2) under the restrictions $\operatorname{det} D \mathbf{u}=1$ and (4.3). To do this, we choose to compute an approximation of the minimizer $\mathbf{u}$ of (4.2) under (4.3) using first order polynomial interpolation on a uniform triangular mesh for the computational domain $[0, R] \times[0, L]$. Then, minimizing the deformation energy amounts to finding the coefficients of $\mathbf{u}$ in the basis of first order Lagrange polynomials. We treat the restriction $\operatorname{det} D \mathbf{u}=1$ locally, that is, it is imposed in each triangle of the mesh, although the numerical algorithm handles this constraint in a relaxed way; see below.

All numerical experiments presented in this section were performed with $L=10, R=0.5$, so as to simulate an elongated bar of aspect ratio 20:1. The number of nodes in the horizontal and vertical directions are $N_{1}=6$ and $N_{2}=111$ respectively, so that the aspect ratio of the triangles of the triangulation is close to 1 . This choice corresponds to a number of 1209 unknowns and 1100 triangles.

We solved the minimization problem using Algencan, a Fortran package for general constrained optimization problems included in Tango (Trustable Algorithms for Nonlinear General Optimization) [25]. The minimization procedure uses the augmented Lagrangian algorithm: an iterative process that uses a shifting strategy to address the constraints. More specifically, a penalty term is added to the objective function, but penalizations apply not to the true constraints, but to the displaced (or shifted) constraints, whose shifting level corresponds to the infeasibility at the solution of the previous iteration. Thus, at each iteration the minimizer, the shift and the penalty parameter are updated, according to rules explained in detail in [8]. The introduction of the shifts prevents the penalty parameter from reaching values so big that the 
objective function would become numerically neglected. Hence, the two quantitites (the objective function and the incompressibility constraints) are maintained in balance. The correction of the shifts can be seen as a step-by-step move towards the feasible region: for the intermediate problems, the constraints are not necessarily satisfied, while the final solution does satisfy the incompressibility constraint in each triangle up to the specified tolerance, being the minimizer of the energy plus a term that penalizes the true constraint (without any shift).

This feature of the augmented Lagrangian method turned out to be crucial for our investigation, as it allowed us to avoid the locking phenomenon occuring when using low-order polynomial approximation under the incompressibility constraint [4]. Indeed, as the approximation space is narrow (since the number of degrees of freedom is only slightly bigger than the number of constraints), the penalty parameter has to be chosen carefully: it has to be big enough to keep the incompressibility constraints satisfied up to the specified tolerance, but if it becomes too big, the penalty term will be dominant in the function to be minimized and the objective function will be neglected. To support this idea, we mention that our first attempt to solve the minimization problem under the incompressibility constraint was by including the constraints in the objective function as a penalty term, with a fixed penalty parameter. In this case we did observe the locking phenomenon: when the penalty parameter was too big, the final deformation was the initial one (that is, the homogeneous deformation). If the penalty parameter was too small, necking was observed, but the incompressibility was lost. However, when adopting an augmented Lagrangian procedure we were able, from the first attempt, to obtain the expected results. Since, as we will see, the experiments reported in Subsections 6.1-6.4 confirm the theoretical results, we consider that the approximation and the minimization algorithm can be trusted.

Nevertheless, we are aware that adopting higher-order approximations [4], saddle-point formulations [9], as well as studying the algorithm convergence under mesh refinement are interesting topics related to our problem. These can all be formulated as future investigation projects.

As for the experiments, we carried them out first for a polyconvex $W$, so as to guarantee the existence of minimizers. The obtained results and our observations are reported in Subsections 6.1-6.3. Then, we show in Subsection 6.4 the output of experiments for which $W$ is not polyconvex. In this section, all figures describing deformations only represent the right-half part of it, since in Section 4 we assumed vertical symmetry.

According to the analysis of Section 3, we differentiated three regions in the parameters $b, c$ :

R1. The assumptions of Lemma 3.3 are satisfied. This corresponds to $b>0$ and $c \geq G(b)$.

R2. The assumptions of Lemma 3.3 do not hold, and (C3) does not hold either. This corresponds to $b>0$ and $-\sqrt{2 b / 3} \leq c<G(b)$.

R3. Assumption (C3) holds. This corresponds to $b>0$ and $F(b) \leq c<-\sqrt{2 b / 3}$.

Cases R1-R2 do not have a natural range of values for the parameter $b$. In contrast, case R3 does, as hinted in Sections 3 and 5. Indeed, the value of $\lambda$ must not be unrealistically high. The percent elongation of a ductile metal typically varies from $25 \%$ to $60 \%$ (corresponding to a value of $\lambda$ from 1.25 to 1.6), while that of rubbers varies from $100 \%$ to $800 \%$ (corresponding to $\lambda$ from 2 to 9 ); see, e.g., [21, Appendix H]. In order to cover a majority of materials, we chose $\lambda$ less than 2 , which corresponds approximately to a value of $b$ less than 1 (see the discussion of case R3 below).

For each of the three regimes, we studied, for all choices of $b, c$ and $\lambda$ :

- the reduction of the elastic energy;

- the maximum width

and the minimum width

$$
\max _{x_{2} \in[0, L]} u^{1}\left(R, x_{2}\right)
$$

$$
\min _{x_{2} \in[0, L]} u^{1}\left(R, x_{2}\right)
$$

of $\mathbf{u}$, as well as their difference;

- the profile of the final deformation. 


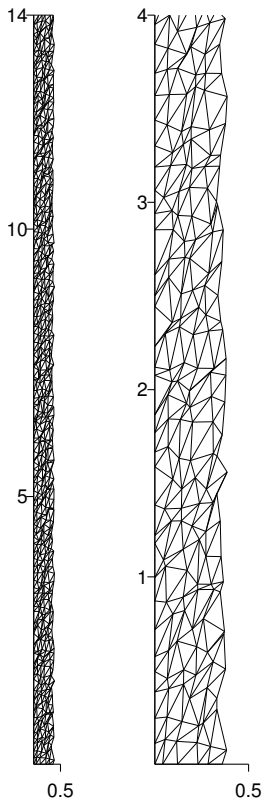

(a) A typical random perturbation $\mathbf{u}_{P}$ of $\mathbf{u}_{H}$.

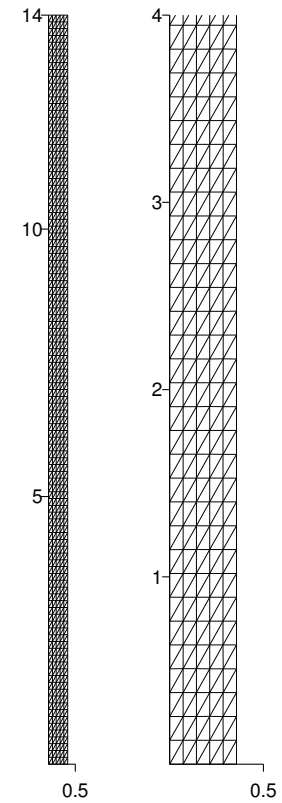

(b) Final deformation $\mathbf{u}$ corresponding to regime $\mathrm{R} 1 ; b=0.2$ (see first line of Table 1, left).

Figure 4: Deformations $\mathbf{u}_{P}$ and $\mathbf{u}$, together with a zoomed part of them; $\lambda=1.4$.

\subsection{Case R1}

Although we know [33] that the minimizer is $\mathbf{u}_{H}$, we performed experiments so as to have an idea of the level of error that the algorithm can make.

Since the deformations $\mathbf{u}_{N}$ and $\mathbf{u}_{C}$ do not apply in this case, the only natural candidate for an initial deformation is $\mathbf{u}_{H}$. The experiments with $\mathbf{u}_{H}$ starting point confirmed that this is also the minimizer.

In order to give the algorithm a chance to escape from $\mathbf{u}_{H}$, we perturbed it. More precisely, we chose the following piecewise affine perturbation: for any node $\mathbf{x}$ of the triangulation which is an unknown of the problem, we define the piecewise affine deformation

$$
\mathbf{u}_{P}(\mathbf{x}):=\mathbf{u}_{H}(\mathbf{x})+\left(\eta^{1}\left(x_{1}\right), \eta^{2}\left(x_{2}\right)\right),
$$

where $\eta^{1}\left(x_{1}\right)$ is a random number uniformly distributed in $\left(-\frac{\lambda^{-1} R}{2 N_{1}}, \frac{\lambda^{-1} R}{2 N_{1}}\right)$ (depending on $x_{1}$ ), and, analogously, $\eta^{2}\left(x_{2}\right)$ is a random number in $\left(-\frac{\lambda L}{2 N_{2}}, \frac{\lambda L}{2 N_{2}}\right)$ (depending on $x_{2}$ ). This choice allows an important deviation of $\mathbf{u}_{P}$ from $\mathbf{u}_{H}$, while keeping low the risk of finding a $\mathbf{u}_{P}$ that reverses the orientation of a triangle. The incompressibility constraint, on the contrary, is lost, but the algorithm will solve that.

We show in Figure 4(a) a typical $\mathbf{u}_{P}$, which naturally looks chaotic.

We list in Table 1 the output of a selection of the experiments performed: we write the values of $\lambda, b$, $c=G(b)$, the ratio $\frac{E}{E\left(\mathbf{u}_{H}\right)}$, where $E$ is the energy of the final deformation $\mathbf{u}$ and the difference between the maximum and the minimum width of the material in the final deformation. Each experiment was performed three times, in order to check whether the outcome was influenced by the different perturbations; we reported in the table the data of the experiment corresponding to the least energy of the deformation.

We observed the following:

- in all experiments the final deformation $\mathbf{u}$ was indistinguishable from the homogeneous deformation $\mathbf{u}_{H}$. As an example, we put in Figure 4(b) the deformation $\mathbf{u}$ corresponding to $\lambda=1.4$ and $b=0.2$; 


\begin{tabular}{l|l|l|l|l}
$\lambda$ & $b$ & $G(b)$ & $E / E\left(\mathbf{u}_{H}\right)$ & diff width \\
\hline 1.4 & 0.2 & -0.2 & 0.993711 & 0.0007 \\
1.5 & 0.4 & -0.395202 & 0.995953 & 0.0004 \\
1.6 & 0.6 & -0.541385 & 0.997666 & 0.0002 \\
1.8 & 0.8 & -0.655178 & 0.999532 & 0.0002
\end{tabular}

\begin{tabular}{l|l|l|l|l}
$\lambda$ & $b$ & $-\sqrt{2 b / 3}$ & $E / E\left(\mathbf{u}_{H}\right)$ & diff width \\
\hline 1.4 & 0.2 & -0.365148 & 1.000146 & 0.0075 \\
1.5 & 0.4 & -0.516398 & 1.000000 & 0.0035 \\
1.6 & 0.6 & -0.632456 & 1.000000 & 0.0054 \\
1.8 & 0.8 & -0.730297 & 1.000000 & 0.0028
\end{tabular}

Table 1: Outcome of the experiments in R1 (left) and R2 (right).

- the difference between the maximum and the minimum width varied from 0.0002 to 0.0007 , which corroborates the conclusion that those deformations cannot be differentiated from $\mathbf{u}_{H}$;

Our conclusion is that an energy ratio of 0.993711 and a difference between the maximum and minimum widths of 0.0007 mean, in practice, that the final deformation is indistinguishable from $\mathbf{u}_{H}$. This shows also that our approximation procedure reproduces the theoretical result established in [33].

Each experiment took very little: fewer than 6 iterations and less than 2 seconds.

\subsection{Case R2}

In case R2 there are no theoretical results: we do not know whether or not the global minimizer is $\mathbf{u}_{H}$. As in case $\mathrm{R} 1$, we chose $\mathbf{u}_{P}$ as a starting point and made three experiments for each value of the parameters. We chose $c=-\sqrt{2 b / 3}$. In Table 1 (right) we list the outcome of a selection of the experiments performed; we wrote that with the least energy in each set of the three experiments. We noticed that:

- as in Case R1, the energy differences were insignificant, although in Case R1 all of them are less than 1, whereas in Case R2 they are greater or equal to 1. We suspect that this is due to Algencan's parameters and stopping criteria.

- the difference between the thickest and thinnest part was always between 0.003 and 0.008 , that is, one order of magnitude higher than in R1.

- all deformations corresponding to the obtained minimizers are visually indistinguishable from the linear deformation, so identical to Figure 4(b) (in the case $\lambda=1.4$ ).

All in all, we do not consider these small differences between the two regimes to be significant. The numerical experiments therefore suggest that $\mathbf{u}_{H}$ is indeed the minimizer, so there may be a chance that the analysis of [33] can be extended to cover this case as well.

Each experiment took fewer than 5 iterations and less than 35 seconds.

\subsection{Case R3}

Case R3 is, of course, the interesting one, and for which the theory of Sections 3 and 5 was developed. We chose $c=F(b)$. In Table 2 (top) we put the parameters common to all experiments: $b, c=F(b), p_{ \pm}$ (see (3.4)), $\lambda_{1}, \lambda_{2}$ (see Figure 3), $R / \lambda_{1}, R / \lambda_{2}$ (maximum and minimum widths of both $\mathbf{u}_{H}$ and $\mathbf{u}_{C}$ ) and $R / \lambda_{1}-R / \lambda_{2}$ (width difference).

We know from the bifurcation analysis mentioned in the introduction that necking can only be observed for $p_{-}<\lambda<p_{+}$; this is corroborated by the equal area rule (Section 5). We mention, however, that proofs of this fact have been only given in simplified models (typically, rod models) and not in the full 2D case. In order to confirm numerically the absence of necking for $\lambda \notin\left(p_{-}, p_{+}\right)$, we did experiments for $\lambda<p_{-}$ and $\lambda>p_{+}$. We did three tries of each experiment starting with $\mathbf{u}_{P}$ : the outcome was very similar to the experiments in R2, in terms of the energy ratio, the difference between the maximum and minimum widths, and also visually (indistinguishable from $\mathbf{u}_{H}$ ). We report in Table 2 (bottom right) the results of the experiments with the least energy among the three tries; we show the ratio between the energy $E$ of the minimizer found and the energy $E\left(\mathbf{u}_{H}\right)$ of the homogeneous deformation. We also show the difference between the maximum and minimum widths. 


\begin{tabular}{l|l|l|l|l|l|l|l|l}
$b$ & $F(b)$ & $p_{-}$ & $p_{+}$ & $\lambda_{1}$ & $\lambda_{2}$ & $R / \lambda_{1}$ & $R / \lambda_{2}$ & $R / \lambda_{1}-R / \lambda_{2}$ \\
\hline 0.3 & -0.460111 & 1.3519 & 1.5683 & 1.27274 & 1.64748 & 0.3939 & 0.3035 & 0.0894 \\
0.4 & -0.531654 & 1.4052 & 1.6581 & 1.31264 & 1.75067 & 0.3809 & 0.2856 & 0.0953 \\
0.5 & -0.594586 & 1.4525 & 1.7367 & 1.34842 & 1.84075 & 0.3708 & 0.2716 & 0.0992 \\
0.6 & -0.651392 & 1.4955 & 1.8073 & 1.38133 & 1.92145 & 0.3620 & 0.2602 & 0.1018 \\
0.7 & -0.703552 & 1.5353 & 1.8718 & 1.41208 & 1.99503 & 0.3541 & 0.2506 & 0.1035
\end{tabular}

\begin{tabular}{l|l|l|l|l|l|l}
$b$ & $\lambda$ & best & $E / E\left(\mathbf{u}_{H}\right)$ & max width & min width & diff width \\
\hline 0.3 & 1.4 & $\mathbf{u}_{N}$ & 0.991119 & 0.3945 & 0.3043 & 0.0902 \\
& 1.5 & $\mathbf{u}_{C}$ & 0.992673 & 0.3904 & 0.3015 & 0.0942 \\
\hline 0.4 & 1.5 & $\mathbf{u}_{N} \mathrm{P}$ & 0.990225 & 0.3834 & 0.2863 & 0.0971 \\
& 1.6 & $\mathbf{u}_{P}$ & 0.993149 & 0.3823 & 0.2870 & 0.0953 \\
\hline 0.5 & 1.6 & $\mathbf{u}_{C} \mathrm{P}$ & 0.989801 & 0.3727 & 0.2682 & 0.1045 \\
& 1.7 & $\mathbf{u}_{P}$ & 0.994733 & 0.3720 & 0.2723 & 0.0997 \\
\hline 0.6 & 1.6 & $\mathbf{u}_{C}$ & 0.990065 & 0.3618 & 0.2601 & 0.1017 \\
& 1.7 & $\mathbf{u}_{P}$ & 0.991793 & 0.3619 & 0.2595 & 0.1024 \\
\hline 0.7 & 1.6 & $\mathbf{u}_{P}$ & 0.991184 & 0.3560 & 0.2506 & 0.1054 \\
& 1.7 & $\mathbf{u}_{N}$ & 0.990258 & 0.3541 & 0.2506 & 0.1035 \\
& 1.8 & $\mathbf{u}_{N} \mathrm{P}$ & 0.993711 & 0.3539 & 0.2505 & 0.1034
\end{tabular}

\begin{tabular}{l|l|l|l}
$b$ & $\lambda$ & $E / E\left(\mathbf{u}_{H}\right)$ & diff width \\
\hline 0.3 & 1.3 & 1.000169 & 0.0032 \\
& 1.6 & 1.000010 & 0.0032 \\
\hline 0.5 & 1.4 & 1.000521 & 0.0082 \\
& 1.8 & 1.000012 & 0.0019
\end{tabular}

Table 2: Experiments in R3. Top: common values of the parameters. Bottom left: Experiments for $\lambda \in\left(p_{-}, p_{+}\right)$. Bottom right: Experiments for $\lambda \notin\left(p_{-}, p_{+}\right)$.

For the case $p_{-}<\lambda<p_{+}$we did experiments with the starting deformations $\mathbf{u}_{P}$ (three tries), $\mathbf{u}_{N}$, perturbation of $\mathbf{u}_{N}$ (three tries), $\mathbf{u}_{C}$ and perturbation of $\mathbf{u}_{C}$ (three tries). The perturbations of $\mathbf{u}_{N}$ and $\mathbf{u}_{C}$ were done in a similar way as $\mathbf{u}_{P}$ was obtained from $\mathbf{u}_{H}$. The algorithm found a final deformation (we call it local minimizer) for each experiment and then we selected the one with the least energy (we call it best minimizer), the data of which is reported in Table 2 (bottom left). Hence, each line of Table 2 (bottom left) describes the best minimizer among 11 local minimizers; we write which initial deformation gave rise to the best minimizer (in column best; we indicate by a $\mathrm{P}$ when it is a perturbation), the ratio between the energy $E$ of the best minimizer and the energy $E\left(\mathbf{u}_{H}\right)$, the maximum and minimum widths, and their difference. Figure 5 show four typical local minimizers found; all local minimizers of this set of experiments look like one of those four. We point out the following observations:

- The best minimizer was always either a smoothed version of $\mathbf{u}_{N}$ (see Figure $5(\mathrm{a})$ ) or an inversion of it (see Figure 5(c)). This agrees with the analysis of [30, Th. 4.4] based in rod theory. It also answers in the affirmative the question posed in [34, p. 316]: necking seems to be the global minimizer for some isotropic homogeneous polyconvex materials out of the class of [33].

- Apart from smoothed versions of $\mathbf{u}_{N}$ (as in Figure 5(a)) and their inversions (see Figure 5(c)), we also found as local minimizers a smoothed version of $\mathbf{u}_{C}$ (thin-thick-thin, as in Figure 5(b)) and an inversion of it (thick-thin-thick, as in Figure 5(d)). The latter two local minimizers seem to contradict the theory of [30, Th. 4.3], although his model and boundary conditions are different from ours.

- Sometimes the initial deformation $\mathbf{u}_{N}$ gave rise to a minimizer that was a smoothed version of $\mathbf{u}_{N}$, but sometimes it gave rise to a minimizer that was an inversion of a smoothed version of $\mathbf{u}_{N}$. Similarly, given the five kinds of initial deformations and the four kinds of local minimizers depicted in Figure 5, almost all of the 20 possibilities (that a particular kind of initial deformation gave rise to a particular kind of local minimizer) were found. Our explanation for this is that, when the initial deformation does not satisfy the incompressibility constraint, in the first iteration the algorithm expands its search to deformations that do satisfy the constraint but are far from the initial one.

- The width difference is considerably bigger than in the previous experiments (Table 1 and Table 2 bottom right). Hence, we can safely say that necking is observed. 


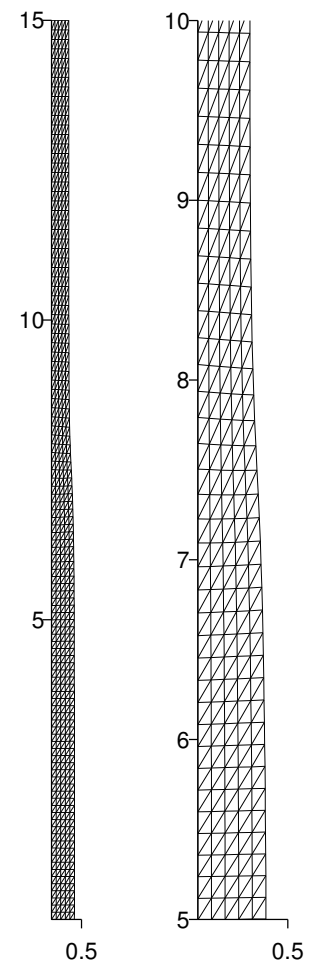

(a) $\lambda=1.5, b=0.4$. Initial deformation: perturbation of $\mathbf{u}_{N} . \quad E / E\left(\mathbf{u}_{H}\right)=0.990225$, diff width $=0.0971$. Best minimizer.

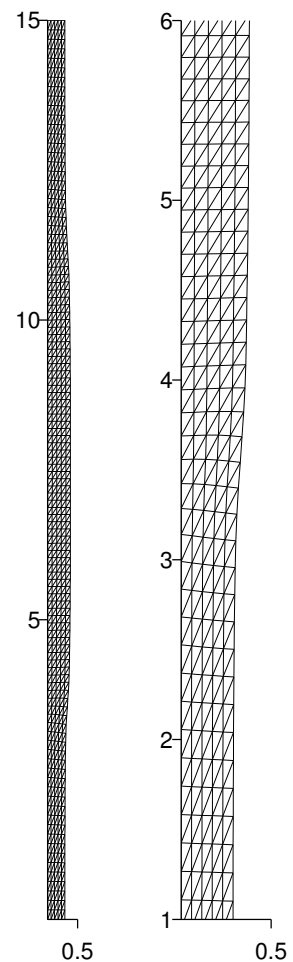

(b) $\lambda=1.5, \quad b=0.4$. Initial deformation: $\mathbf{u}_{C}$. $E / E\left(\mathbf{u}_{H}\right)=0.992609$, diff width $=0.0929$.
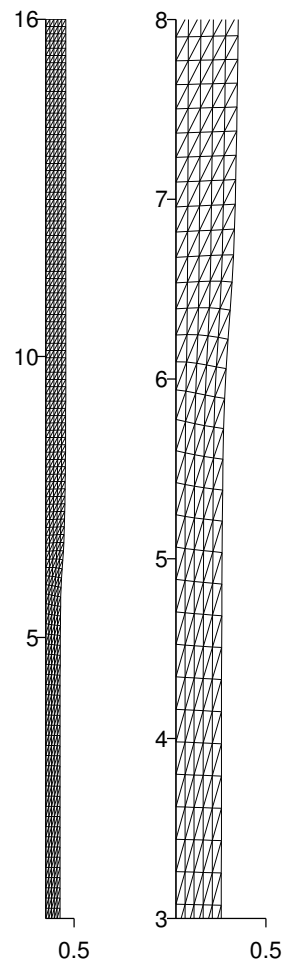

(c) $\lambda=1.6, \quad b=0.7$. Initial deformation: $\mathbf{u}_{P}$ $E / E\left(\mathbf{u}_{H}\right)=0.991184$, diff width $=0.1054$. Best minimizer.

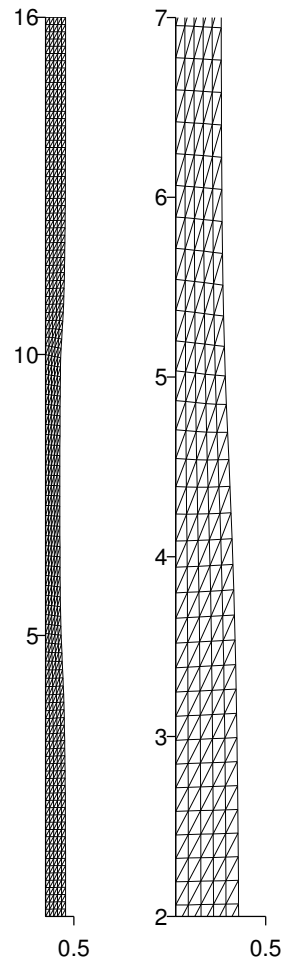

(d) $\lambda=1.6, \quad b=0.7$. Initial deformation: $\mathbf{u}_{H}$. $E / E\left(\mathbf{u}_{H}\right)=0.993071$, diff width $=0.1010$

Figure 5: Experiments in R3. Four different types of local minimizers, together with a zoomed part of them.

- The energy ratio $E / E\left(\mathbf{u}_{H}\right)$ is definitively smaller than in the experiments of R2 (see Table 1, right) and those of R3 for $\lambda \notin\left(p_{-}, p_{+}\right)$(see Table 2, bottom right), and also smaller than those of R1 (see Table 1, left).

- Comparing $R / \lambda_{1}$ and $R / \lambda_{2}$ of Table 2 (top) with the maximum and minimum widths of Table 2 (bottom left), as well as the difference of these quantities, we can see how well the equal area rule predicts the maximum and minimum widths of the minimizer.

Each experiment in R3 took less than 2 minutes and fewer than 8 iterations.

\subsection{Experiments not in the regimes R1-R3.}

These experiments correspond to lack of polyconvexity, which happens when $b \leq 0$ or when $b>0$ and $c<F(b)$. Without polyconvexity, the existence of minimizers is not guaranteed. Since analyzing lack of polyconvexity is not the aim of this study, we only did four experiments: $\mathbf{u}_{H}$ as a starting point and three tries with $\mathbf{u}_{P}$. In order to allow for a fair comparison with the experiments already done, we chose $\lambda=1.4$, $b=0.4$ and $c=-0.6$ (compare with Table 2). We noticed the following:

- With $\mathbf{u}_{H}$ as a starting point, the algorithm was not able to find a minimizer within 50 iterations: it was the first time that this occurred. 

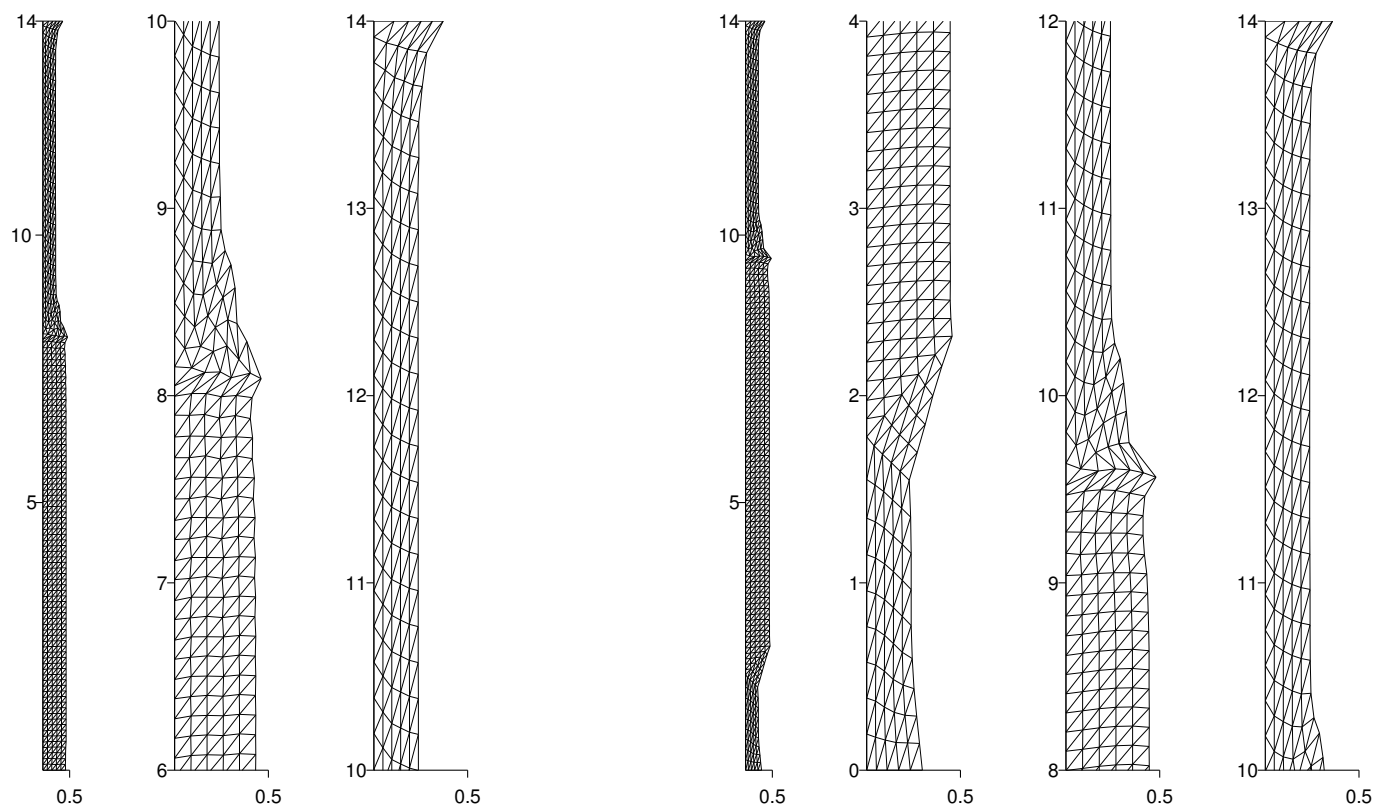

Figure 6: Experiments with $\lambda=1.4, b=0.4$ and $c=-0.6$ (regime of lack of polyconvexity), with starting point $\mathbf{u}_{P}$, together with zoomed parts of them. First experiment: three pictures on the left; second experiment: four pictures on the right.

- In one of the three tries with $\mathbf{u}_{P}$ as a starting point, the algorithm did not converge; in the other two the algorithm converged: one with $E / E\left(\mathbf{u}_{H}\right)=0.680375$ (see Figure 6, three pictures on the left) and other with $E / E\left(\mathbf{u}_{H}\right)=0.679656$ (see Figure 6, four pictures on the right). As one can see, there is a drastic reduction in the energy. This seems to suggest that an extra energy coming from inelastic phenomena must be taken into account; this is corroborated by the fact that, in general, lack of polyconvexity cannot model elasticity [6]. This phenomenom should be analyzed within the context of loss of ellipticity (see, e.g., [39]), but this study lies outside the scope of this work.

- Visually, the pictures of Figure 6 remind necking (in fact, half-necking and centred necking, respectively), but the deformations are rougher.

The experiments that converged took less than 2 minutes and fewer than 9 iterations, so in the range of the experiments in R3.

\section{Conclusions}

We present a summary of the tasks performed as well as the conclusions we can draw from them.

- We described a systematic procedure to construct 2D polyconvex, incompressible, homogeneous, isotropic stored-energy functions for hyperleastic materials exhibiting necking.

- The linearly elastic behaviour of those materials is compatible with any linearly elastic behaviour.

- We constructed a quartic polynomial $\varphi$ depending on two parameters such that $\varphi^{\prime}$ represents the force (on the ends of the sample bar) as a function of the extension, and that the stored-energy function $W(\mathbf{F})$ equals $\varphi(\nu(\mathbf{F}))$, where $\nu(\mathbf{F})$ is the largest singular value of the deformation gradient $\mathbf{F}$.

- We characterized polyconvexity and other important properties of $W$ in terms of $\varphi$ and its parameters. 
- Within polyconvexity, we distinguished three regimes in the parameters of $\varphi$ : in regime R1, the assumptions of [33] holds; in regime R2, the assumptions of [33] do not hold and $\varphi^{\prime}$ is increasing; in regime $\mathrm{R} 3, \varphi^{\prime}$ is not always increasing.

- We did experiments with five types of initial deformations: a random perturbation of the linear deformation $\mathbf{u}_{H}$, an idealized half-neck deformation, a perturbation of it, an idealized centred-neck deformation and a perturbation of it.

- Numerics confirmed that in regime R1 the minimizer is $\mathbf{u}_{H}$, as proved in [33]. Numerics suggested that in regime $\mathrm{R} 2$ the minimizer is $\mathbf{u}_{H}$, although no proof has been given. In regime R3, we calculated $\lambda_{1}<\lambda_{2}$ according to the equal area rule; when $\lambda \notin\left(\lambda_{1}, \lambda_{2}\right)$, the numerical minimizer was $\mathbf{u}_{H}$; when $\lambda \in\left(\lambda_{1}, \lambda_{2}\right)$, the global minimizer showed a half-necking, and some local minimizers showed centrednecking. This fits the theory of necking based on rod models, even though our case is a full 2D model.

- Polyconvexity is essential for the numerical algorithm and the model. In the absence of polyconvexity, the algorithm either does not converge or shows a minimizer suggesting an inelastic phenomenom.

\section{Acknowledgements}

The authors wish to thank Welington de Oliveira for the suggestion of using Algencan. C.M.-C. has been supported by Project MTM2014-57769-C3-1-P and the "Ramón y Cajal" programme RYC-2010-06125 of the Spanish Ministry of Economy and Competitivity, and the ERC Starting grant no. 307179. M. S. was supported by the Basque Government through the BERC 2014-2017 programme and by the Spanish Ministry of Economy and Competitivity through the BCAM Severo Ochoa excellence accreditation SEV-2013-0323.

\section{References}

[1] S. S. Antman, Nonuniqueness of equilibrium states for bars in tension, J. Math. Anal. Appl., 44 (1973), pp. 333349 .

[2] _ Nonlinear problems of elasticity, vol. 107 of Applied Mathematical Sciences, Springer, New York, second ed., 2005.

[3] S. S. Antman And E. R. CARbone, Shear and necking instabilities in nonlinear elasticity, J. Elasticity, 7 (1977), pp. 125-151.

[4] I. BABUŠKA AND M. SuRI, Locking effects in the finite element approximation of elasticity problems. Technical Note BN-1119, Institute for Physical Science and Technology, Maryland.

[5] J. M. BALL, Solid mechanics lecture notes. University of Oxford.

[6] Convexity conditions and existence theorems in nonlinear elasticity, Arch. Ration. Mech. Anal., 63 (1977), pp. 337-403.

[7] — Differentiability properties of symmetric and isotropic functions, Duke Math. J., 51 (1984), pp. 699-728.

[8] E. G. Birgin And J. M. Martínez, Practical Augmented Lagrangian Methods for Constrained Optimization, vol. 10 of Fundamental of Algorithms, SIAM, Philadelphia, first ed., 2014.

[9] D. Braess And P. Ming, A finite element method for nearly incompressible elasticity problems, Math. Comp., 74 (2005), pp. 25-52 (electronic).

[10] J. Carr, M. E. Gurtin, And M. Slemrod, Structured phase transitions on a finite interval, Arch. Rational Mech. Anal., 86 (1984), pp. 317-351.

[11] Y.-C. Chen, Non-axisymmetric necking, Matematiche (Catania), 46 (1991), pp. 493-502. VI International Conference on Waves and Stability in Continuous Media (Acireale, 1991).

[12] P. G. Ciarlet, Mathematical elasticity. Vol. I, vol. 20 of Studies in Mathematics and its Applications, NorthHolland Publishing Co., Amsterdam, 1988. 
[13] B. D. Coleman, Necking and drawing in polymeric fibers under tension, Arch. Rational Mech. Anal., 83 (1983), pp. 115-137.

[14] B. Dacorogna, Direct methods in the calculus of variations, Springer, New York, second ed., 2008.

[15] H.-H. DAI AND Q. BI, On constructing the unique solution for the necking in a hyper-elastic rod, J. Elasticity, 82 (2006), pp. 215-241.

[16] G. DeL Piero, Lower bounds for the critical loads of elastic bodies, J. Elasticity, 10 (1980), pp. $135-143$.

[17] G. Del Piero and R. Rizzoni, Weak local minimizers in finite elasticity, J. Elasticity, 93 (2008), pp. $203-244$.

[18] J. L. ERICKSEn, Equilibrium of bars, J. Elasticity, 5 (1975), pp. 191-201. Special issue dedicated to A. E. Green.

[19] - Introduction to the thermodynamics of solids, vol. 131 of Applied Mathematical Sciences, Springer-Verlag, New York, revised ed., 1998.

[20] Y. B. Fu, Perturbation methods and nonlinear stability analysis, in Nonlinear elasticity: theory and applications (Y. B. Fu and R. W. Ogden, eds.), vol. 283 of London Math. Soc. Lecture Note Ser., Cambridge Univ. Press, Cambridge, 2001, pp. 345-391.

[21] J. M. Gere And B. J. Goodno, Mechanics of materials, Cengage Learning, Stamford, CT, 8th ed., 2013.

[22] M. E. Gurtin, An introduction to continuum mechanics, Academic Press Inc., New York, 1981.

[23] R. Hill And J. W. Hutchinson, Bifurcation phenomena in the plane tension test, J. Mech. Phys. Solids, 23 (1975), pp. 239-264.

[24] J. Kristensen, On conditions for polyconvexity, Proc. Amer. Math. Soc., 128 (2000), pp. $1793-1797$.

[25] J. M. MarTínez, Tango project. http://www.ime.usp.br/ egbirgin/tango/index.php.

[26] A. Mielke, Hamiltonian and Lagrangian flows on center manifolds, vol. 1489 of Lecture Notes in Mathematics, Springer-Verlag, Berlin, 1991.

[27] — Necessary and sufficient conditions for polyconvexity of isotropic functions, J. Convex Anal., 12 (2005), pp. 291-314.

[28] C. Mora-Corral, Explicit energy-minimizers of incompressible elastic brittle bars under uniaxial extension, C. R. Math. Acad. Sci. Paris, 348 (2010), pp. 1045-1048.

[29] R. W. Ogden, Nonlinear elastic deformations, Ellis Horwood Series: Mathematics and its Applications, Ellis Horwood Ltd., Chichester; Halsted Press; John Wiley \& Sons, New York, 1984.

[30] N. Owen, Existence and stability of necking deformations for nonlinearly elastic rods, Arch. Rational Mech. Anal., 98 (1987), pp. 357-383.

[31] P. Rosakis, Characterization of convex isotropic functions, J. Elasticity, 49 (1998), pp. 257-267.

[32] M. ŠILHAVÝ, On semiconvexity properties of rotationally invariant functions in two dimensions, Czechoslovak Math. J., 54(129) (2004), pp. 559-571.

[33] J. Sivaloganathan And S. J. Spector, On the global stability of two-dimensional, incompressible, elastic bars in uniaxial extension, Proc. R. Soc. A, 466 (2010), pp. 1167-1176.

[34] _ - On the stability of incompressible elastic cylinders in uniaxial extension, J. Elasticity, 105 (2011), pp. 313330.

[35] — On the global stability of compressible elastic cylinders in tension, J. Elasticity, 120 (2015), pp. 161-195.

[36] S. J. Spector, On the absence of bifurcation for elastic bars in uniaxial tension, Arch. Rational Mech. Anal., 85 (1984), pp. 171-199.

[37] J. Sylvester, On the differentiability of $\mathrm{O}(n)$ invariant functions of symmetric matrices, Duke Math. J., 52 (1985), pp. 475-483.

[38] Z. Wesolowski, Stability in some cases of tension in the light of the theory of finite strain, Arch. Mech. Stos., 14 (1962), pp. 875-900.

[39] L. Zee And E. Sternberg, Ordinary and strong ellipticity in the equilibrium theory of incompressible hyperelastic solids, Arch. Rational Mech. Anal., 83 (1983), pp. 53-90. 